\title{
Hesitant fuzzy TOPSIS method and its application in the cloud resource scheduling
}

\author{
Dong-Ke Yang \\ Institute of Quality Development, Kunming University of \\ Science and Technology \\ KUST \\ Kunming, China
}

\begin{abstract}
CRS) is according to users' needs for service to pick out an appropriate resource, but restricted by the actual situation, it is needed to obtain several acceptable solutions for users to choose, the paper puts forward a kind of TOPSIS method based on hesitant fuzzy sets for cloud resource scheduling; Firstly hesitant fuzzy elements describe all considered cloud resources' attribute values and attribute weights, and then define a new hesitant fuzzy distance measure, finally propose a new hesitant fuzzy closeness to sort cloud resources. Finally use a cloud resource scheduling example to test feasibility and effectiveness of new method.
\end{abstract}

Keywords-Cloud resource scheduling; hesitant fuzzy sets; TOPSIS; distance measure; closeness

\section{INTRODUCTION}

Cloud computing is a kind of calculation based on the Internet, and using virtualization, instantaneous deployment, and broadband network will be a large number of key technologies such as virtual and dynamic resources through the Internet to realize connectivity, communication and interoperability of resources, and it in the form of Internet service is provided to the user application, thus under cloud environment resource scheduling is very complicated.

Existing cloud resource scheduling algorithm according to different mission requirements of tasks for the user to select a virtual machine resources, such as heuristic task scheduling algorithm, multiple resource pools scheduling algorithm, load balance scheduling algorithm, random integer planning scheduling algorithm[1-9]. Such as in [1] the cloud resource scheduling algorithm based on genetic algorithm is put forward, through heuristic intelligent algorithm, for user tasks in addition to complete the time or the minimum average task completion time of the virtual machine. Due to the business characteristics of cloud computing, in [4-7] scheduling algorithm was designed. In [9] proposed a cloud resource scheduling strategy based on expectancy theory, through quantitative user's QoS requirements user tasks assigned to the most appropriate cloud resources. The above work studied from various aspects the cloud resource allocation strategy, but ignored the user's hesitate psychology, and focused on service quality effect's global optimization after service aggregation, not emphasized local service quality, thus by these methods polymerase chain structure of cloud service is the optimal

\author{
Ding-Hong Peng* \\ Institute of Quality Development, Kunming University of \\ Science and Technology \\ KUST \\ Kunming, China \\ pengdinghong2006@163.com
}

single solution satisfying the constraint conditions rather than an acceptable solution [10-12].

TOPSIS [13] is a kind of classic methods to solve the problem of multi-attribute decision making, Original TOPSIS method just deals with multiple attribute decision making problems when the attribute weight values and attribute values are both accurate number, in fact affected by their own experience, knowledge, etc., the evaluation of decision maker is not easy to determine [14-21], thus there are a lot of literatures to solve the problem of this respect, fuzzy set theory [22] is born to describe the uncertain psychological of decision makers. For the above problems, this paper will put forward a kind of hesitant fuzzy TOPSIS method, for resolving the difficulty that the precise value of membership degree is not easy to determine and more accurate to simulate the complex psychology of decision makers, so using the expanded form of fuzzy sets, hesitant fuzzy elements[23,24], and through the new definition of hesitant fuzzy distance measure and relative closeness to sort various cloud resources, we can select more than one acceptable solutions.

\section{HESITANT FUZZY SET AND ITS OPERATIONS}

Hesitant fuzzy sets (HFSs) were first introduced by Torra [23] and Torra and Narukawa [24], it permits the membership degree of an element to a set to be represented as several possible values between 0 and 1 . HFSs are very useful in dealing with the situations where people have hesitancy in providing their preferences over objects in a decision-making process.

Definition 1. Let $M=\left\{\mu_{1}, \ldots, \mu_{N}\right\}$ be a set of membership functions. Then, the hesitant fuzzy set [24] associated with $M$, that is $h_{M}$, is defined as follows:

$$
h_{M}(x)=\bigcup_{\mu \in M}\{\mu(x)\}
$$

Definition 2. Let $h, h_{1}$ and $h_{2}$ are three hesitant fuzzy elements, part of the algorithm between hesitant fuzzy elements [25] as follows:
(1) $\lambda h=\bigcup_{\gamma \in h}\left\{1-(1-\gamma)^{\lambda}\right\}$
(2) $h^{c}=\bigcup_{\gamma \in h}\{1-\gamma\}$ 
(3) $h_{1} \bigcup h_{2}=\bigcup_{\gamma_{1} \in h_{1}, \gamma_{2} \in h_{2}}\left\{\max \left(\gamma_{1}, \gamma_{2}\right)\right\}$

(4) $h_{1} \oplus h_{2}=\bigcup_{n \in h_{1} \cdot r_{2}=l_{2}}\left\{\gamma_{1}+\gamma_{2}-\gamma_{1} \gamma_{2}\right\}$

Definition 3. Let $h$ is a hesitant fuzzy element, $s(h)=\frac{1}{l(h)} \sum_{\gamma \in h} \gamma$ is the scoring function of $h$ [25], where $l(h)$ is the number of values in $h$. For two hesitant fuzzy element $h_{1}$ and $h_{2}$, if $s\left(h_{1}\right)>s\left(h_{2}\right)$, then $h_{1}>h_{2}$; if $s\left(h_{1}\right)=s\left(h_{2}\right)$, then $h_{1}=h_{2}$.

Definition 4. Let $h_{1}$ and $h_{2}$ are two hesitant fuzzy elements, then the distance measure between $h_{1}$ and $h_{2}$ is defined as $d\left(h_{1}, h_{2}\right)$, which satisfies the following properties [25]:

(1) $0 \leq d\left(h_{1}, h_{2}\right) \leq 1$;

(2) $d\left(h_{1}, h_{2}\right)=0$ If and only if $h_{1}=h_{2}$;

(3) $d\left(h_{1}, h_{2}\right)=d\left(h_{2}, h_{1}\right)$.

\section{TOPSIS METHOD UNDER COMPLETELY HESITANT FUZZY ENVIRONMENT}

$\mathrm{Xu}$ and Xia [25] proposed for hesitant fuzzy sets Euclidean distance, Hamming distance and etc. requiring two corresponding hesitant fuzzy elements in two hesitant fuzzy sets must be the same, the previous practice is subjective to add some membership value to keep the same length, but this way lead to repeating and the distortion of information, so it is necessary to find a hesitant fuzzy distance without subjectively adding membership degree value.

Definition 5. Let $h_{1}$ and $h_{2}$ are three hesitant fuzzy elements, then the GAM distance measure between $h_{1}$ and $h_{2}$ is

$$
\left.d_{G A M}\left(h_{1}, h_{2}\right)=\frac{1}{2} \underset{\rightarrow}{\rightarrow}\left(h_{1}, h_{2}\right)+\underset{\rightarrow}{d}\left(h_{2}, h_{1}\right)\right)
$$

Where

$$
\begin{aligned}
& \underset{\rightarrow}{d}\left(h_{1}, h_{2}\right)=\frac{1}{\# h_{1}} \sum_{\gamma_{1} \in h_{1}} \sqrt[\# h_{2}]{\prod_{\gamma_{2} \in h_{2}}\left|\gamma_{1}-\gamma_{2}\right|} \\
& \underset{\rightarrow}{d}\left(h_{2}, h_{1}\right)=\frac{1}{\# h_{2}} \sum_{\gamma_{2} \in h_{2}} \sqrt[\# h_{1}]{\prod_{\gamma_{1} \in h_{1}}\left|\gamma_{1}-\gamma_{2}\right|}
\end{aligned}
$$

Where $\# h_{1}$ and $\# h_{2}$ are number of real Numbers in $h_{1}$ and $h_{2}$.

The following Theorem 1 shows that new hesitant fuzzy distance measure meets axiomatic definition of hesitant fuzzy distance measure.

Theorem 1. Let $h_{1}$ and $h_{2}$ are two hesitant fuzzy elements, then the GAM distance measure between $h_{1}$ and $h_{2}$ meets axiomatic Definition 4 of hesitant fuzzy distance measure.

Proof

(1) Obviously $0 \leq d_{G A M}\left(h_{1}, h_{2}\right) \leq 1$;
(2) When $h_{1}=h_{2}, \prod_{\gamma_{2} \in h_{2}}\left|\gamma_{1}-\gamma_{2}\right|=0 \quad$, then $\quad d_{G A M}\left(h_{1}, h_{2}\right)=0$; when $d_{G A M}\left(h_{1}, h_{2}\right)=0$, then $\prod_{\gamma_{2} \in h_{2}}\left|\gamma_{1}-\gamma_{2}\right|=0$;

(3) Obviously $d\left(h_{1}, h_{2}\right)=d\left(h_{2}, h_{1}\right)$.

Based on a hesitant fuzzy GAM distance, we give an extended TOPSIS when criterion value and the attribute weights are hesitant fuzzy elements. Specific steps are following:

Step 1 : Hesitant fuzzy decision matrix

Assume that a certain set of decision scheme for decisionmaking problem $A=\left\{A_{1}, A_{2}, \cdots, A_{n}\right\}$, where $A_{i}$ is the ith alternative; The attribute set of decision scheme is $X=\left\{x_{1}, x_{2}, \cdots, x_{m}\right\}$, where $x_{j}$ is the $j$ th attribute, $A_{i}$ can be represented by a set $A_{i}=\left\{\left\langle x_{j}, \tilde{h}_{A_{i}}\left(x_{j}\right)\right\rangle \mid x_{j} \in X\right\}$, where $\tilde{h}_{A_{i}}\left(x_{j}\right)=\left\{\gamma \mid \gamma \in \tilde{h}_{A_{i}}\left(x_{j}\right), 0 \leq \gamma \leq 1\right\}, i=1,2, \ldots, m ; j=1,2, \ldots, n$. $\tilde{h}_{A_{i}}\left(x_{j}\right)$ is the first project in the first rule of possible membership degree, our shorthand $\tilde{h}_{i j}$ for them. Therefore, hesitant fuzzy decision matrix is following :

$\tilde{H}=\left[\begin{array}{cccc}\tilde{h_{11}} & \tilde{h_{12}} & \cdots & \tilde{h_{1 m}} \\ \tilde{h_{21}} & \tilde{h_{22}} & \cdots & \tilde{h_{2 m}} \\ \vdots & \vdots & \ddots & \vdots \\ \tilde{h_{n 1}} & \tilde{h_{n 2}} & \cdots & \tilde{h_{n m}}\end{array}\right]$

$\tilde{W}=\left\{\tilde{w}_{1}, \tilde{w}_{2}, \cdots, \tilde{w}_{m}\right\}$ is the hesitant fuzzy weight vector of attributes, where $\tilde{w}_{j}=\bigcup_{\tilde{\gamma}_{j} \in w_{j}}\left\{\tilde{\gamma}_{j}\right\}$.

Attributes are divided into benefit and cost type, when the rule is cost type, we can use a hesitant fuzzy complementary set $\tilde{h_{i j}^{c}}$ to standardize the attribute value; when a rule is benefit type, $\tilde{h}_{i j}$ remain the same. So we get standardized matrix $\tilde{H}^{\prime}$ :

$$
\tilde{H}=\left[\begin{array}{cccc}
\tilde{\gamma}_{11} & \tilde{\gamma}_{12} & \cdots & \tilde{\gamma}_{1 m} \\
\tilde{\gamma_{21}} & \tilde{\gamma}_{22} & \cdots & \tilde{\gamma}_{2 m} \\
\vdots & \vdots & \ddots & \vdots \\
\tilde{\gamma_{n 1}} & \tilde{\gamma}_{n 2} & \cdots & \tilde{\gamma}_{n m}
\end{array}\right]
$$

Step 2 : Define the positive and negative ideal solution (PIS and NIS) (17):

$$
\tilde{A^{+}}=\left\{\tilde{A_{1}^{+}}, \tilde{A_{2}^{+}}, \ldots, \tilde{A_{j}^{+}}, \ldots, \tilde{A_{m}^{+}}\right\}
$$


Where $\tilde{A_{j}^{+}}=\bigcup_{\alpha_{i j} \in \gamma_{i j}, i=1,2, \ldots, n} \max \left\{\alpha_{i j}\right\}, j=1,2, \cdots, m$

$\tilde{A^{-}}=\left\{\tilde{A_{1}^{-}}, \tilde{A_{2}^{-}}, \ldots, \tilde{A_{j}^{-}}, \ldots, \tilde{A_{m}^{-}}\right\}$

Where $\tilde{A_{j}^{-}}=\bigcup_{\alpha_{i j} \in \bar{Y}_{i j}, i=1,2, \ldots, n} \min \left\{\alpha_{i j}\right\}, j=1,2, \cdots, m$

Step 3 : Calculate the separation measures $\tilde{d}_{i}^{+}$and $\tilde{d}_{i}^{-}$of each alternative $A_{i}$ from the hesitant fuzzy PIS $\tilde{A}^{+}$and the hesitant fuzzy NIS $\tilde{A}^{-}$, respectively.

$$
\begin{aligned}
& \tilde{d_{i}^{+}}=\bigoplus_{j=1}^{m} d_{G A M}\left(\tilde{\gamma}_{i j}, \tilde{A_{j}^{+}}\right) \tilde{w}_{j}
\end{aligned}
$$

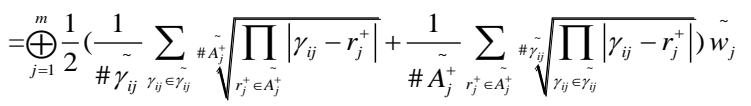

(8)

$$
\begin{aligned}
& \tilde{d}_{i}^{-}=\bigoplus_{j=1}^{m} d_{G A M}\left(\tilde{\gamma}_{i j}, \tilde{A_{j}^{-}}\right) \tilde{w}_{j} \\
& =\bigoplus_{j=1}^{m} \frac{1}{2}\left(\frac{1}{\# \tilde{\gamma_{i j}}} \sum_{\gamma_{i j} \in \tilde{\gamma}_{i j}} \underset{A A_{j}}{\prod_{r_{j}^{-} \in \tilde{A}_{j}^{-}}\left|\gamma_{i j}-r_{j}^{-}\right|}+\frac{1}{\# \tilde{A_{j}^{-}}} \sum_{r_{j}^{-} \in \tilde{A}_{j}^{-}} \# \sqrt{\# \gamma_{i j}} \sqrt{\prod_{\gamma_{i j} \in \gamma_{i j}}\left|\gamma_{i j}-r_{j}^{-}\right|}\right) \tilde{w_{j}}
\end{aligned}
$$

Where $\# \tilde{\gamma}_{i j}, \# \tilde{M}_{j}^{+}$and $\# \tilde{M}_{j}^{-}$are number of real Numbers in $\tilde{\gamma}_{i j}$, $\tilde{M}_{j}^{+}$and $\tilde{M}_{j}^{-}, \tilde{w}_{j}$ is hesitant fuzzy criterion weight.

Step 4 : Calculate the closeness coefficients to the ideal solution for each alternative

Firstly through scoring function to compare $\tilde{d}_{i}^{+}$and $\tilde{d}_{i}^{-}$, then according to the following function to sort all the alternatives

$$
C_{i}^{*}=1-\frac{1}{1+\sqrt{d_{H M}\left(\tilde{\left.D_{i}^{-}, \tilde{D}_{i}^{+}\right)}\right.}}
$$

If $\tilde{d}_{i}^{-}$is larger than $\tilde{d}_{i}^{+}, C_{i}^{*}$ is better to be larger; if $\tilde{d}_{i}^{+}$is larger than $\tilde{d}_{i}^{-}, C_{i}^{*}$ is better to be smaller.

Obviously, $0 \leq C_{i}^{*} \leq 1, i=1,2, \ldots, n$ when $C_{i}^{*}$ is closer to 1 , it means that $A_{i}$ is closer the positive ideal solution and further away from the negative ideal solution. Therefore according to the degree value to rank all alternatives, the maximum value is the optimal solution.

\section{A CLOUD RESOURCE SCHEDULING EXAMPLE}

The cloud resource scheduling model is a $n * t$ resource scheduling model composed of $n$ virtual machine and $t$ user tasks. Firstly Virtual machine resources collection $V=\left\{A_{1}, A_{2}, \cdots, A_{i}, \cdots, A_{n}\right\}$ consists of $n$ virtual machine resources mutually independent between the various resources, and virtual machine resource $v m_{i}$ including $m$ different attributes, here we consider three attributes: the virtual machine CPU, Memory and Bandwidth properties, and they can be described by $C_{1}, C_{2}$ and $C_{3}$ respectively, and there are four virtual machine resources. Because decision makers' evaluation will be affected by their knowledge level, experience and character which can lead to the difference between the evaluation information. To clearly reflect different expert opinion, attribute values and attribute weight are described by hesitant fuzzy element, as shown in TABLE 1 and TABLE 2.

TABLE I. HESITANT FUZZY DECISION MATRIX

\begin{tabular}{|l|l|l|l|}
\hline & \multicolumn{1}{c|}{$\boldsymbol{C 1}$} & \multicolumn{1}{c|}{$\boldsymbol{C 2}$} & \multicolumn{1}{c|}{$\boldsymbol{C 3}$} \\
\hline A1 & $\{0.9,0.6,0.4\}$ & $\{0.8,0.4,0.3,0.1\}$ & $\{0.9,0.8,0.4\}$ \\
\hline A2 & $\{0.7,0.5,0.4,0.3\}$ & $\{0.7,0.4\}$ & $\{0.9,0.7,0.5,0.3\}$ \\
\hline A3 & $\{0.7,0.5,0.4,0.2\}$ & $\{0.8,0.6,0.3\}$ & $\{0.9,0.8,0.6,0.4,0.2\}$ \\
\hline A4 & $\{0.9,0.8,0.6\}$ & $\{0.8,0.5,0.3\}$ & $\{0.8,0.5,0.3\}$ \\
\hline
\end{tabular}

TABLE II. HESITANT FUZZY ATTRIBUTE WEIGHTS

\begin{tabular}{|c|c|c|}
\hline $\boldsymbol{C 1}$ & $\boldsymbol{C 2}$ & $\boldsymbol{C 3}$ \\
\hline$\{0.7,0.4\}$ & $\{0.5,0.3,0.2\}$ & $\{0.8,0.6\}$ \\
\hline
\end{tabular}

With the new methods proposed in this paper to solve the problem:

Step 1: Because of the three evaluation attributes for efficiency, so the decision data in table 1 don't need to standard, decision matrix as follows:

$H^{\prime}=\left[\begin{array}{ccc}\{0.9,0.6,0.4\} & \{0.8,0.4,0.3,0.1\} & \{0.9,0.8,0.4\} \\ \{0.7,0.5,0.4,0.3\} & \{0.7,0.4\} & \{0.9,0.7,0.5,0.3\} \\ \{0.7,0.5,0.4,0.2\} & \{0.8,0.6,0.3\} & \{0.9,0.8,0.6,0.4,0.2\} \\ \{0.9,0.8,0.6\} & \{0.8,0.5,0.3\} & \{0.8,0.5,0.3\}\end{array}\right]$

Step 2: Obtain the positive and negative ideal solution (PIS and NIS):

$$
\begin{aligned}
& A^{+}=\{\{0.9,0.8,0.7\},\{0.9,0.8,0.7\},\{0.9,0.8\}\} \\
& A^{-}=\{\{0.6,0.5,0.4,0.3,0.2\},\{0.5,0.4,0.3,0.1\},\{0.6,0.4,0.3,0.2\}\}
\end{aligned}
$$

Step 3: Calculate the separation measures ${\tilde{d_{i}^{+}}}^{+}$and $\tilde{d}_{i}^{-}$of each alternative $A_{i}$ from the hesitant fuzzy PIS $\tilde{A}^{+}$and the hesitant fuzzy NIS $\tilde{A}^{-}$, respectively.

$$
\begin{aligned}
& d_{1}^{+}=\left\{\begin{array}{l}
0.2012,0.1825,0.1805,0.1778,0.1773,0.1677, \\
0.1614,0.1586,0.1581,0.1565,0.1561,0.1532
\end{array}\right\} \\
& d_{2}^{+}=\left\{\begin{array}{l}
0.1894,0.1791,0.175,0.1699,0.1642,0.1626, \\
0.1593,0.1551,0.1536,0.152,0.1493,0.1478
\end{array}\right\} \\
& d_{3}^{+}=\left\{\begin{array}{l}
0.2043,0.1908,0.1871,0.1853,0.1805,0.1733, \\
0.169,0.1678,0.1666,0.1628,0.161,0.1549
\end{array}\right\}
\end{aligned}
$$




$$
\begin{aligned}
d_{4}^{+} & =\left\{\begin{array}{l}
0.2473,0.2429,0.2302,0.2257,0.2233,0.2212, \\
0.2188,0.2167,0.2035,0.1989,0.1979,0.1964
\end{array}\right\} \\
d_{1}^{-} & =\left\{\begin{array}{l}
0.1547,0.1521,0.151,0.1411,0.14,0.1384, \\
0.1373,0.1372,0.1362,0.1261,0.1234,0.1223
\end{array}\right\} \\
d_{2}^{-} & =\left\{\begin{array}{l}
0.1411,0.1373,0.1342,0.1304,0.1242,0.1204, \\
0.1171,0.1133,0.1008,0.0969,0.0936,0.0897
\end{array}\right\} \\
d_{3}^{-} & =\left\{\begin{array}{l}
0.1706,0.167,0.1626,0.159,0.1383,0.1382, \\
0.1345,0.1344,0.13,0.1299,0.1263,0.1261
\end{array}\right\} \\
d_{4}^{-} & =\left\{\begin{array}{l}
0.2116,0.1933,0.1912,0.1885,0.1865,0.179, \\
0.1724,0.1697,0.1675,0.1655,0.1627,0.16
\end{array}\right\}
\end{aligned}
$$

Step 4 : Calculate the closeness coefficients to the ideal solution for each alternative

TABLE III. CLOSENESS COEffiCIENTS

\begin{tabular}{|c|c|c|c|}
\hline$C_{1}^{*}$ & $C_{2}^{*}$ & $C_{3}^{*}$ & $C_{4}^{*}$ \\
\hline 0.5537 & 0.6038 & 0.5544 & 0.5494 \\
\hline
\end{tabular}

Because $C_{2}^{*}>C_{3}^{*}>C_{1}^{*}>C_{4}^{*}$, so the best choice is $A_{2}$, the second choice is $A_{3}$, the third choice is $A_{1}$. Presented in this paper, the method provides the optimal and suboptimal solution for the user to choose, so the method has a wider application range than ever before for the cloud resource scheduling.

\section{CONCLUSIONS}

The paper puts forward a kind of TOPSIS method based on hesitant fuzzy sets for cloud resource scheduling; Firstly hesitant fuzzy elements describe all considered cloud resources' attribute values and attribute weights, which can express the users' preferences and can be directly obtained, and then defines a new hesitant fuzzy distance measure, which can overcome the problem of subjectively adding membership value in existing interval hesitate fuzzy distance measures, finally propose a new hesitant fuzzy closeness to sort cloud resources, avoiding disadvantages of simple way that using scoring function to handling blur.

\section{ACKNOWLEDGMENT}

The work was partly supported by the national natural science fund project (61364016), China postdoctoral science foundation of China (2015T80990 and 2014M550473)and applied basic research projects of Yunnan province (2014 FB136).

\section{REFERENCES}

[1] Zhao C, Zhang S, Liu Q, et al. Independent tasks scheduling based on genetic algorithm in cloud computing[C]// International Conference on Wireless Communications, NETWORKING and Mobile Computing. IEEE Press, 2009:5548-5551.

[2] Cao J, Spooner D P, Jarvis S A, et al. Agent-Based Grid Load Balancing Using Performance-Driven Task Scheduling[J]. In Proceedings of the 17th IEEE International Parallel and Distributed Processing Symposium (IPDPS 2003, 2003:49--58.

[3] Buyya R, Abramson D, Giddy J, et al. Economic models for resource management and scheduling in Grid computing $[\mathrm{J}]$. Concurrency \& Computation Practice \& Experience, 2002, 14(13-15):1507-1542.
[4] Nimis J, Anandasivam A, Borissov N, et al. SORMA - Business Cases for an Open Grid Market: Concept and Implementation[M]// Grid Economics and Business Models. Springer Berlin Heidelberg, 2008:173184.

[5] Younge A J, Laszewski G V, Wang L, et al. Efficient resource management for Cloud computing environments[C]// International Conference on Green Computing. IEEE Computer Society, 2010:357364.

[6] Song H, Yang S, Wu B, et al. An Optimal Algorithm for Scheduling Tasks within Deadline and Budget Constraints[C]// Fifth International Joint Conference on Inc, Ims and IDC. IEEE Computer Society, 2009:62-65.

[7] Buyya R, Murshed M, Abramson D, et al. Scheduling parameter sweep applications on global Grids: a deadline and budget constrained costtime optimization algorithm[J]. Software Practice \& Experience, 2010, 35(5):491-512.

[8] Dirk Neumann, Jochen Stößer, Christof Weinhardt. Bridging the Adoption Gap - Developing a Roadmap for Trading in Grids[J]. 2008, 18(1):65-74.

[9] Ping Q I, Long-Shu L I. Task Scheduling Algorithm Based on Fuzzy Quotient Space Theory in Cloud Environment[J]. Journal of Chinese Computer Systems, 2013, 34(8):1793-1797.

[10] Wang Y, Dai G P, Hou Y R. Dynamic methods of trust - A ware composite service selection[J]. Chinese Journal of Computers, 2009, 32(8):1668-1675.

[11] Maurer M, Emeakaroha V C, Brandic I, et al. Cost-benefit analysis of an SLA mapping approach for defining standardized Cloud computing goods[J]. Future Generation Computer Systems, 2011, 28(1):39-47.

[12] Su S, Fei L, Yang F C. Iterative selection algorithm for service composition in distributed environments[J]. Science in China, 2008, 51(11):1841-1856.

[13] Tzeng G H, Huang J J. Multiple attribute decision making: methods and applications[M]. CRC press, 2011.

[14] Chu T C, Lin Y C. A fuzzy TOPSIS method for robot selection[J]. International Journal of Advanced Manufacturing Technology, 2003, 21(4):284-290.

[15] Ashtiani B, Haghighirad F, Makui A, et al. Extension of fuzzy TOPSIS method based on interval-valued fuzzy sets[J]. Applied Soft Computing, 2009, 9(2):457-461.

[16] Lan R, Fan J L. TOPSIS decision-making method for three parameters interval-valued fuzzy sets[J]. Systems Engineering - Theory \& Practice, 2009, 29(5):129-136.

[17] Peng D H, Gao C Y, Gao Z F. Generalized hesitant fuzzy synergetic weighted distance measures and their application to multiple criteria decision-making[J]. Applied Mathematical Modelling, 2013, 37(8):5837-5850.

[18] Mokhtarian M N, Sadi-Nezhad S, Makui A. A new flexible and reliable IVF-TOPSIS method based on uncertainty risk reduction in decision making process[J]. Applied Soft Computing, 2014, 23(5):509-520.

[19] Wang Y J. The evaluation of financial performance for Taiwan container shipping companies by fuzzy TOPSIS[J]. Applied Soft Computing,2014,22:28-35.

[20] Wang J Q, Wang D D, Zhang H Y, et al. Multi-criteria outranking approach with hesitant fuzzy sets[J]. Or Spectrum, 2014, 36(4):10011019.

[21] Yang C, Chen W, Peng D H. An Approach based on TOPSIS for Interval Type-2 Fuzzy Multiple Attributes Decision-making[J]. International Journal of Control \& Automation, 2015, 8.

[22] Zadeh L A. Fuzzy sets [J]. Information \& Control, 1965, 8(3):338-353.

[23] Torra V. Hesitant fuzzy sets[J]. International Journal of Intelligent Systems, 2010, 25(6):529-539.

[24] Torra V, Narukawa Y. On hesitant fuzzy sets and decision[C]// IEEE International Conference on Fuzzy Systems, 2009. Fuzz-Ieee. IEEE, 2009:1378-1382.

[25] Xu Z, Xia M. Distance and similarity measures for hesitant fuzzy sets[J]. Information Sciences, 2011, 181(11):2128-2138. 